\title{
Orbit Ephemeris Failure Detection in a GNSS Regional Application
}

\author{
Jongsun Ahn* and Young Jae Lee**
}

Konkuk University, 120 Neundong-ro, Gwangin-gu, Seoul 143-701, South Korea

\author{
Dae Hee Won*** \\ University of Colorado at Boulder, 431 UCB, Boulder, CO 80309, USA
}

\section{Hyang-Sig Jun**** and Chanhong Yeom*****}

Korea Aerospace Research Institute, 169-84 Gwahang-no, Yuseong-gu, Daejeon 305-806, South Korea

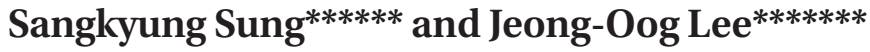 \\ Konkuk University, 120 Neundong-ro, Gwangin-gu, Seoul 143-701, South Korea
}

\begin{abstract}
To satisfy civil aviation requirements using the Global Navigation Satellite System (GNSS), it is important to guarantee system integrity. In this work, we propose a fault detection algorithm for GNSS ephemeris anomalies. The basic principle concerns baseline length estimation with GNSS measurements (pseudorange, broadcasted ephemerides). The estimated baseline length is subtracted from the true baseline length, computed using the exact surveyed ground antenna positions. If this subtracted value differs by more than a given threshold, this indicates that an ephemeris anomaly has been detected. This algorithm is suitable for detecting Type A ephemeris failure, and more advantageous for use with multiple stations with various long baseline vectors.

The principles of the algorithm, sensitivity analysis, minimum detectable error (MDE), and protection level derivation are described and we verify the sensitivity analysis and algorithm availability based on real GPS data in Korea. Consequently, this algorithm is appropriate for GNSS regional implementation.
\end{abstract}

Key words: GNSS, Integrity, Ephemeris Failure Detection, Baseline Length Estimation

\section{Introduction}

The Global Navigation Satellite System (GNSS) is used to compute user navigation solutions with certain accuracy at any time and location of interest. With increasing GNSS applications, the civil aviation community has been trying to implement primary navigation systems using GNSS. To ensure aircraft safety in the civil aviation implementation of GNSS, the implemented system must meet integrity requirements.

Research on various GNSS implemented systems and on the use of ground facilities, additional satellites, and navigation sensors, has been conducted to address integrity issues [1] [2]. The main line of integrity issues includes threat definition, development of detection algorithms within time to alert, and the protection level (PL) for confidence in the user navigation solution. One of the threats facing GNSS involves orbit ephemerides, which are generated periodically at a ground control facility (a GPS Operation Control Segment) and are transmitted to the user by satellites [3]. The frequency of the ephemeris anomaly tends to decrease, however. Because the ramifications for user position error is critical, the system must include monitoring and detection processing [4][5].
This is an Open Access article distributed under the terms of the Creative Commons Attribution Non-Commercial License (http://creativecommons.org/licenses/by$\mathrm{nc} / 3.0 /$ ) which permits unrestricted non-commercial use, distribution, and reproduction in any medium, provided the original work is properly cited. (c)

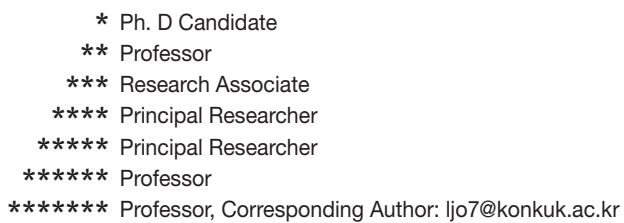


Ephemeris anomalies are conventionally divided into two groups (Types A and B) with respect to the occurrence of satellite maneuvers. Type $A$ is an ephemeris failure event during the process of a satellite maneuver, whereas Type B can be issued in ephemeris generation or transmission but no satellite maneuver is involved [6].

Various methods have been proposed for the detection of both Type A and Type B ephemeris anomalies. Type B events can be detected by examining the consistency between the broadcast ephemeris and prior validated ephemeris. The magnitude of detectable satellite position error due to a Type B anomaly depends on the validated time of a prior ephemeris. Representative algorithms include the ephemeris-ephemeris test, YE-TE test, and almanacephemeris test. They compute satellite position and monitor consistency of results within a given threshold [7]. However, these algorithms have the limitation of requiring a validated prior ephemeris, and hence cannot detect Type A ephemeris failure. On the other hand, Type A failure is relatively more difficult to detect than Type B because there is no validated prior ephemeris to compare with the ephemeris of a satellite just after an orbit maneuver. One method of detecting Type A failure is to monitor the range measurement correction (pseudorange correction, PRC) derived from the broadcast ephemeris and the location of the ground station antenna [7]. Other methods estimate satellite position error with range measurements [8][9] or estimate the differential range of ground stations with short baseline vectors and range measurements [10][13]. However, these algorithms have some weaknesses. First, when the direction of satellite position error is orthogonal to the line-of-sight, ephemeris failure on test statistics is small. Next, there is the limitation of baseline length (short baseline, 100-400 m), and using carrier phase measurement that must resolve integer ambiguity.

To supplement these algorithms, we propose a detection algorithm using non-limited baseline length and code measurement for Type A failures. This methodology, based on the law of cosines in trigonometry, estimates the baseline lengths of multiple ground antennas.

We describe the methodology in more detail in the following sections. In section 2 , we introduce the concept of the algorithm, describe test statistics and a threshold, analyze sensitivity [14] between test statistics and ephemeris failure, and derive the ephemeris protection level (EPL) and MDE [15] for algorithm availability. In section 3, we focus on evaluating the algorithm described above using real GPS data. So, we evaluated sensitivity analysis and availability performance using MDE and ephemeris protection level for landing aircraft using real GPS data of multiple reference stations (RSs) in Korea. Finally, we describe the conclusions and future work in section 4 .

\section{Baseline Length Estimation Algorithm}

This section introduces the concept of the proposed algorithm (test statistics and threshold), analyses the sensitivity of test statistics according to satellite position error due to ephemeris failure, and derives the MDE, which is the performance parameter of fault detection, and the ephemeris protection level.

\subsection{Principle of the Algorithm}

The detection methodology estimates the baseline length between reference station (RS) antennas using range measurements of RS and the broadcast ephemeris to be validated. The baseline length is assumed to be error-free because RS antenna location is computed accurately and precisely. In this section, we describe the estimation method for single baseline length based on two RSs. According to the First Cosine Law, one side length of triangle can be computed using the other two sides' length and their induced angle. In Fig. (1), this law can be applied in GNSS implementation. One side of the triangle represents the length of the displacement vector $\left(\hat{\vec{x}}_{A B}\right)$ and the other sides' lengths can be determined with range measurements $\left(\rho_{A}^{i}, \rho_{B}^{i}\right)$ received by two RSs. The induced angle is then computed by the unit vector $\left(\hat{\vec{x}}_{A B}\right)$ of the baseline and unit vectors $\left(\vec{e}_{A}^{i}, \vec{e}_{B}^{i}\right)$ corresponding to the premeasured antenna locations of RSs and the broadcast ephemeris of the satellite [14].

$$
\left\|\vec{x}_{A B}\right\|=\rho_{A}^{i} \times \frac{\vec{e}_{A}^{i} \cdot \vec{e}_{A B}^{T}}{\left\|\vec{e}_{A}^{i}\right\| \vec{e}_{A B}^{T} \|}-\rho_{B}^{i} \times \frac{\vec{e}_{B}^{i} \cdot \vec{e}_{A B}^{T}}{\left\|\vec{e}_{B}^{i}\right\| \vec{e}_{A B}^{T} \|}=\rho_{A}^{i} \times\left(\vec{e}_{A}^{i} \cdot \vec{e}_{A B}^{T}\right)-\rho_{B}^{i} \times\left(\vec{e}_{B}^{i} \cdot \vec{e}_{A B}^{T}\right)
$$

The test statistic (TS) is defined by the following equation (2), computed by subtracting the estimated baseline length $\left\|\hat{\vec{x}}_{A B}\right\|$ from the premeasured one $\left(\left\|\vec{x}_{A B}\right\|\right)$.

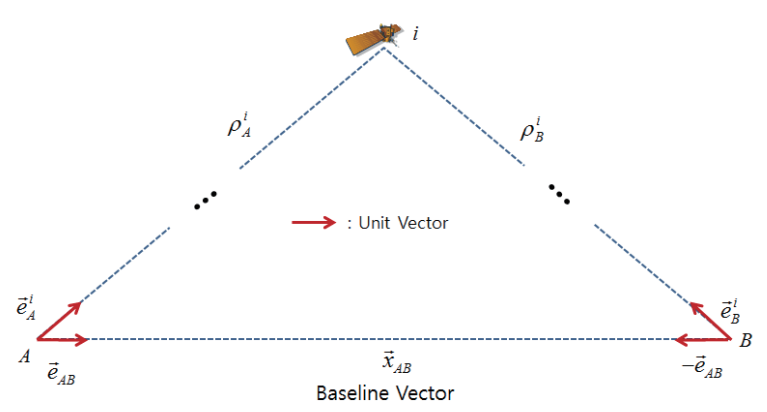

Fig. 1. Geometry condition of two RS antennas (A, B) and a satellite i. 


$$
T S_{A B}^{i}=\left\|\vec{x}_{A B}\right\|-\left\|\hat{\vec{x}}_{A B}\right\|
$$

As shown in equation (1), the test statistic's variation depends on the range measurements and the induced angle. The range measurements are assumed to be fault-free and are not treated further in this work. We focus on the test statistic's variation due to induced angle error resulting from broadcast ephemeris failure.

However, range measurement errors in the ephemeris under normal conditions must be accounted for in the test statistic's accuracy. The range measurement errors are defined as

$$
\begin{aligned}
& \rho_{u}^{i}=R_{u}^{i}+I^{i}+T^{i}+c\left(b_{u}-b^{i}\right)+\varepsilon_{u}^{i} \\
& R_{u}^{i}: \text { True range }(\mathrm{m}) \\
& I^{i}: \text { Ionosphere delay error }(\mathrm{m}) \\
& T^{i}: \text { Troposphere delay error }(\mathrm{m}) \\
& b_{u}: \text { Receiver clock error }(\mathrm{s}) \\
& b^{i}: \text { Satellite clock error }(\mathrm{s}) \\
& \varepsilon_{u}^{i}: \text { Noise, multipath, etc. } \\
& c: \text { Speed of light }(\mathrm{m} / \mathrm{s})
\end{aligned}
$$

Several methods are introduced to mitigate part of the error in range measurement. First, a model-based mitigation method is proposed for ionosphere delay and troposphere delay error. The range differential method then estimates range errors by subtracting the true range from the range measurements (also called pseudorange correction). The receiver clock error is also reduced by subtraction of range measurements between satellites on common RS (called the double difference between satellites). As described above, the combination of subtraction of receivers and satellites gives the best performance from the perspective of relative range measurement accuracy.

However, in this work, a model-based mitigation method is used to mitigate range measurement errors arising from the ionosphere and troposphere. The receiver clock bias is estimated by computation of the user's position in a process using the weighted least squares method. Despite the adverse effect on accuracy of range measurements, we recognized that combining single and double difference methods posed a contradiction in this algorithm because these methods use the broadcast ephemeris (to be validated by test statistics). The test statistics and validated ephemeris must be independent for reliable ephemeris fault detection. In addition, the required range is not a relative value, but an absolute measurement. The methods for mitigating range measurement errors are summarized in Table 1.

The threshold for ephemeris failure detection is determined by the probability property of the test statistics and system continuity requirements. We assume that test statistics follow a Gaussian distribution [11] with a zero mean, and that the continuity requirement (LAAS CAT-I, which is the probability of false alarm in case of the first rising of the day) is $1.9 \times 10^{-4}\left(K_{F F A}=3.73\right)$ [12]. The threshold (TH) is also designed with respect to satellite elevation for navigation integrity and continuity, according to equation (4). Because test statistics are affected by corrected range measurements, the threshold has a stringent boundary as satellite elevation increases relative to the case of a satellite at low elevation.

$$
T H_{T S}^{i}(\theta)=\mu_{T S}^{i}(\theta) \pm\left(K_{F F A} \times \sigma_{T S}^{i}(\theta)\right)
$$

$\mu_{T S}^{i}:$ Test statistics mean $(\mathrm{m})$

$\sigma_{T S}^{i}:$ Standard deviation of test statistics $(\mathrm{m})$

$\theta$ : Elevation angle (degrees)

$K_{F F A}$ :Sigma multiplier based on the continuity requirement

\subsection{Sensitivity Analysis}

Various geometry conditions can be formed between ground baseline vectors and satellites. Consequently, the detection performance of this algorithm depends on the geometry condition. In this section, we derive the relationship between satellite position error and the test statistics and analyze the properties of this algorithm with regard to geometry [14].

In equation (5) and Fig. 2, we define $\vec{e}_{A}^{i, f}, \vec{e}_{B}^{i, f}$ to be unit line-of-sight (LOS) vectors from two RS antennas (A, B) to the satellite i when ephemeris failure occurs. $\vec{e}_{A}^{i}$ and $\vec{e}_{B}^{i},, \rho^{i}$ is geometry range between RS and the satellite $i$, and $\delta \vec{R}^{i}$ represents the faulty vector of satellite $i[13]$.

Table 1. Mitigation methods for range measurement error.

\begin{tabular}{|l|l|}
\hline Error components & Mitigation Methods \\
\hline Ionospheric delay & $\begin{array}{l}\text { Klobuchar model } \\
\text { (with GPS Navigation Message Iono. Parameters) }\end{array}$ \\
\hline Troposheric delay & Sasstamonien model \\
\hline Satellite / Receiver clock & Broadcast ephemeris / Weighted least square \\
\hline
\end{tabular}




$$
\begin{aligned}
\vec{e}_{A}^{i, f} & =\vec{e}_{A}^{i}+\delta \vec{e}_{A}^{i}, \quad \vec{e}_{B}^{i, f}=\vec{e}_{B}^{i}+\delta \vec{e}_{B}^{i} \\
\delta \vec{e}^{i} & =\frac{\left(I-\vec{e}^{i, T} \cdot \vec{e}^{i}\right) \cdot \delta \vec{R}^{i}}{\rho^{i}}
\end{aligned}
$$

Substituting equation (5) into equation (2) yields equation (6).

$$
\begin{aligned}
& T S_{A B}^{i}=\delta \vec{R}^{i, T} \cdot\left[\vec{e}_{A}^{i, T} \cdot \vec{e}_{A}^{i}-\vec{e}_{B}^{i, T} \cdot \vec{e}_{B}^{i}\right]^{T} \cdot \vec{e}_{A B} \\
& =\delta \vec{R}^{i, T} \cdot\left[E_{A B}^{i}\right]^{T} \cdot \vec{e}_{A B} \\
& \vec{e}_{A B} \text { : Unit baseline vector }\left(\vec{x}_{A B}\right) \\
& E_{A B}^{i}=\vec{e}_{A}^{i, T} \cdot \vec{e}_{A}^{i}-\vec{e}_{B}^{i, T} \cdot \vec{e}_{B}^{i}
\end{aligned}
$$

Equation (6) can be simplified in local coordinates that use the baseline vector as the $x$-axis. Fig. (3) shows the local coordinates and the geometry of the satellite.

In local coordinates, $\vec{e}_{A}^{i}$ and $\vec{e}_{B}^{i}$ can be defined as in equation (7) with local elevation angle $(\theta)$ and local azimuth angle $(\psi)$.

$$
\begin{aligned}
& \vec{e}_{A}^{i}=\cos \theta_{A} \cos \psi_{A} \hat{i}_{1}+\cos \theta_{A} \sin \psi_{A} \hat{i}_{2}+\sin \theta_{A} \hat{i}_{3} \\
& \vec{e}_{B}^{i}=\cos \theta_{B} \cos \psi_{B} \hat{i}_{1}+\cos \theta_{B} \sin \psi_{B} \hat{i}_{2}+\sin \theta_{B} \hat{i}_{3}
\end{aligned}
$$

In equation (7), the local elevation angles $\left(\theta_{A}, \theta_{B}\right)$ from reference stations $(A, B)$ can be assumed as similar $\left(\theta_{A} \approx \theta_{B}\right)$,

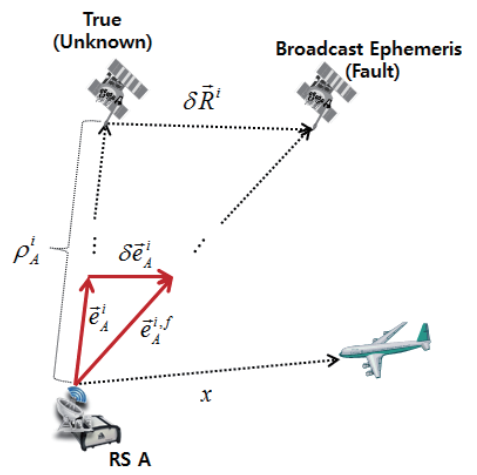

Fig. 2. Geometry of ephemeris fault condition.

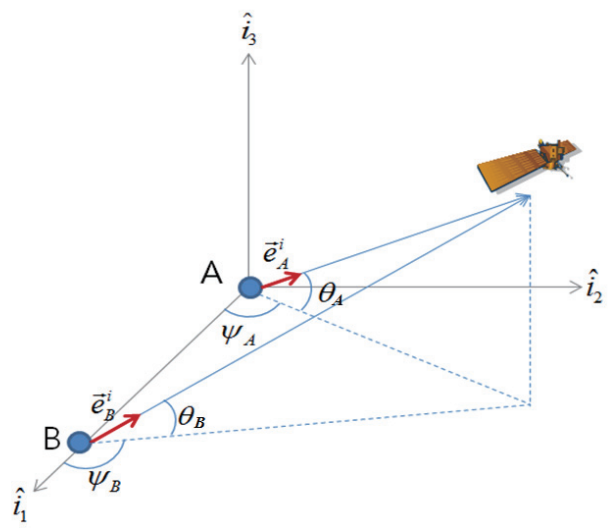

Fig. 3. Local coordinates. because range from GNSS satellite and reference (over 20,000 $\mathrm{km}$ ) is quite large relative to the baseline length between two reference stations. If this assumption is adopted, triangle (reference $A, B$, and satellite) can be assumed to be an isosceles triangle. Using this geometry property the local elevation angles $\left(\theta_{A}, \theta_{B}\right)$ are expressed as $\theta$ regardless of reference position.

Using equation (7) in equation (6), $E_{A B}^{i}$ becomes

$$
\left(E_{A B}^{i}\right)^{T}=\left[\begin{array}{ccc}
\cos ^{2} \theta\left(\cos ^{2} \psi_{A}-\cos ^{2} \psi_{B}\right) & \cos ^{2} \theta\left(\sin \psi_{A} \cos \psi_{A}-\sin \psi_{B} \cos \psi_{B}\right) & \sin \theta \cos \theta\left(\cos \psi_{A}-\cos \psi_{B}\right) \\
\cos ^{2} \theta\left(\cos \psi_{A} \sin \psi_{A}-\cos _{B} \sin \psi_{B}\right) & \cos ^{2} \theta\left(\sin ^{2} \psi_{A}-\sin ^{2} \psi_{B}\right) & \sin \theta \cos \theta\left(\sin \psi_{A}-\sin \psi_{B}\right) \\
\cos \theta \sin \theta\left(\cos \psi_{A}-\cos \psi_{B}\right) & \cos \theta \sin \theta\left(\sin \psi_{A}-\sin \psi_{B}\right) & 0
\end{array}\right] \text { (8) }
$$

The baseline unit vector of equation (6) can be derived as equation (9) [10],

$$
\vec{e}_{A B}=\left[\begin{array}{lll}
1 & 0 & 0
\end{array}\right]^{T}
$$

and substituting equation (9) into equation (6) yields [18]

$$
\begin{aligned}
& T S_{A B}^{i}=\left(E_{A B}^{i}\right)_{L}^{T} \cdot \delta \vec{R}^{i} \\
& \left(E_{A B}^{i}\right)_{L}=\left[E_{A B}^{i}\right]^{T} \cdot \vec{e}_{A B} \\
& \left(E_{A B}^{i}\right)_{L}^{T}=\left[\begin{array}{c}
\cos ^{2} \theta\left(\cos ^{2} \psi_{A}-\cos ^{2} \psi_{B}\right) \\
\cos ^{2} \theta\left(\cos \psi_{A} \sin \psi_{A}-\cos \psi_{B} \sin \psi_{B}\right) \\
\cos \theta \sin \theta\left(\cos \psi_{A}-\cos \psi_{B}\right)
\end{array}\right]^{T}
\end{aligned}
$$

Equation (10) shows the sensitivity relationship between test statistics and the fault in satellite location resulting from ephemeris failure. When the Schwarz inequality is applied in equation (10), we can derive the sensitivity relation in the worst-case geometry condition.

$$
\left\|T S_{A B}^{i}\right\| \leq\left\|\left(E_{A B}^{i}\right)_{L}^{T}\right\|\left\|\delta \vec{R}^{i}\right\|
$$

This shows that the sensitivity increases when the local elevation angle and the azimuth angle are close to $0^{\circ}$ and $90^{\circ}$, respectively. Accordingly, given these properties, this algorithm is sufficient for fault detection for rising satellites of low elevation angle. From a local azimuth perspective, various baseline vectors are required using multiple RS and baseline length should be extended to increase the satellite's induced angle. This means that the algorithm above is applicable for wide-area implementations of GNSS such as Space-Based Augmentation System (SBAS) and Ground Regional Augmentation System (GRAS) rather than narrowarea implementations.

\subsection{Ephemeris Protection Level and MDE}

GNSS implementations conduct a two-step process 
to ensure navigation integrity. First, the information of faulty satellites, identified using fault detection algorithms, is broadcast to the user within a limited time; then, an airborne user computes the protection level corresponding to the available satellites' geometry used to compute the navigation solution. The protection level is computed based on various hypotheses and compared with the allowable position error bound, known as the alert limit (AL), to check GNSS implementation availability [12][16].

In this section we derived the vertical PL $\left(\mathrm{VPL}_{\mathrm{He}}\right)$, which estimates the impact of one satellite failure (undetected at a ground facility) on an airborne user's position, and MDE, based on the proposed ephemeris fault detection algorithm. MDE is used to compute the P Value, broadcast as integrity parameters to the user together with the pseudorange correction (PRC). To derive a $\mathrm{VPL}_{\mathrm{He}}$, an estimated user's conservative position error can be shown in equation (13) using the Cauchy-Schwarz inequality [12].

$$
\left|\delta X_{\text {vert }}^{i}\right| \leq\left|S_{\text {vert }}^{i}\right|\left[\frac{\left\|\delta R^{i}\right\|}{\rho_{A}^{i}}\right]\|x\|+\left|\sum_{i=1}^{n} S_{\text {vert }}^{i} \delta \rho_{\text {others }}^{i}\right|
$$

where $S_{v e r t}^{i}$ is the vertical component of the projection matrix of the range error source onto the position error and $\delta \rho_{\text {others }}^{i}$ is nominal range measurement error (ionosphere, troposphere, multipath, receiver noise, etc.)

$\left\|\delta R^{i}\right\|$ is the satellite position error due to the undetected ephemeris failure, but in a real case, $\left\|\delta R^{i}\right\|$ is hard to figure out using $T S^{i}$ in equation (2). So, we assume that worst case of ephemeris failure detection processor has occurred, the same as algorithm threshold. Then, equation (4) is substituted into the worst case of equation (12), and the satellite position error is derived with equation (14).

$$
\left\|\delta \vec{R}^{i}\right\|=\frac{\mu_{T S}^{i} \pm\left(K_{F F A} \times \sigma_{T S}^{i}\right)}{\left\|\left(E_{A B}^{i}\right)_{L}^{T}\right\|}
$$

When affection of nominal condition of test statistics is considered, the equation (13) is derived as equation (15). We assume that the test statistics correspond to a Gaussian distribution with zero mean and standard deviation $\sigma_{T S}^{i}$.

$$
\left|\delta X_{\text {vert }}^{i}\right| \leq\left|S_{\text {vert }}^{i}\left[\frac{K_{F F A} \times \sigma_{T S}^{i}}{\rho_{A}^{i}\left\|\left(E_{A B}^{i}\right)_{L}^{T}\right\|}\right]\|x\|+\right| S_{\text {vert }}^{i}\left[\frac{T S^{i}}{\rho_{A}^{i}\left\|\left(E_{A B}^{i}\right)_{L}^{T}\right\|}\right]\|x\|+\sum_{i=1}^{n} S_{\text {vert }}^{i} \delta \rho_{\text {others }}^{i} \mid
$$

The second and third terms on the right hand side of equation (15) are developed into equation (16).

$$
\begin{aligned}
& \sqrt{\left(S_{\text {vert }}^{i}\right)^{2}\left[\frac{\sigma_{T S}^{i}}{\rho_{A}^{i}\left\|\left(E_{A B}^{i}\right)_{L}^{T}\right\|}\right]^{2}(\|x\|)^{2}+\sum_{i=1}^{n}\left(S_{\text {vert }}^{i}\right)^{2}\left(\sigma_{\text {others }}^{i}\right)^{2}} \\
& \leq\left|S_{\text {vert }}^{i}\right|\left[\frac{\sigma_{T S}^{i}}{\rho_{A}^{i}||\left(E_{A B}^{i}\right)_{L}^{T} \|}\right]\|x\|+\sqrt{\sum_{i=1}^{n}\left(S_{\text {vert }}^{i}\right)^{2}\left(\sigma_{\text {others }}^{i}\right)^{2}}
\end{aligned}
$$

where $\sigma_{\text {others }}^{i}$ is the standard deviation of $\delta \rho_{\text {others }}^{i}$. Equation (16) is substituted into equation (15) and applying the $K_{M D}$ associated with the probability of missed detection, the protection level is derived as equation (17)

$$
V P L_{H e}^{i}=\left|S_{\text {vert }}^{i}\right|\left[\frac{\left(K_{F F A}+K_{M D}\right) \times \sigma_{T S}^{i}}{\rho_{A}^{i}\left\|\left(E_{A B}^{i}\right)_{L}^{T}\right\|}\right]\|x\|+K_{M D} \sqrt{\sum_{i=1}^{n}\left(S_{\text {vert }}^{i}\right)^{2}\left(\sigma_{\text {others }}^{i}\right)^{2}}
$$

The MDE of the proposed algorithm, shown in equation (18), is defined in the first term on the right hand side of equation (17). According to the integrity requirement, the MDE of the fault detection algorithm is a confidential value corresponding to probability of integrity risk. The P-Value $\left(P_{A}^{i}\right)$, derived using the MDE, is one of the integrity parameters broadcast to the user. The P-Value represents the decorrelation due to ephemeris error between the RS and the aircraft [19].

$$
\begin{aligned}
& M D E^{i}=\frac{\left(K_{F F A}+K_{M D}\right) \times \sigma_{T S}^{i}}{\left\|\left(E_{A B}^{i}\right)_{L}^{T}\right\|} \\
& P_{A}^{i}=M D E^{i} / \rho_{A}^{i}
\end{aligned}
$$

As shown in equation (18), the MDE of the proposed algorithm depends on the norm of the worst-case sensitivity vector, which fluctuates with the geometry condition. However, the previous algorithms use a fixed MDE as the P-Value. Equation (19) is substituted into equation (17); then the EPL of the proposed algorithm is derived in equation (21) [17].

$$
\begin{aligned}
& V P L_{H e}^{i}=\left|S_{\text {vert }}^{i}\right| P_{A}^{i}|\| x|+K_{M D} \sqrt{\sum_{i=1}^{n}\left(S_{\text {vert }}^{i}\right)^{2}\left(\sigma_{\text {others }}^{i}\right)^{2}} \\
& V P L_{A, \text { eph }}=\max \left(V P L_{A, \text { eph }}^{i}\right)
\end{aligned}
$$

The derived VPLHe applies the geometry condition and is expected to ensure integrity and increase availability. Further details and validation are described in the following sections. 


\section{Experiment and Results}

In this section, we evaluate the proposed algorithm using real GPS data for multiple reference stations. First, sensitivity equation (Section 2.1), is evaluated with respect to geometry conditions (local elevation angle, local azimuth, baselines length), and then we compute the threshold based on test statistics. Finally, we check the applicability of algorithm using MDE and EPL in case of landing aircraft at Gimpo International Airport.

\subsection{Sensitivity Evaluation Results}

To evaluate the sensitivity of the proposed algorithm with respect to ephemeris failure, we compare the norm of the worst-case sensitivity vector with that of a similar algorithm based on short baseline vectors and range measurements. Using real GPS data, we present a simulation for verification of sensitivity properties.

The relevant algorithm for comparison uses a short baseline and carrier phase measurement. The reason for its selection is that its information (range measurement, baseline, broadcast ephemeris, etc.) for generation of test statistics is analogous to the proposed algorithm and its detection properties also depend on the geometry condition between the baseline vector and the satellites.

Figure 4 shows the norm of the sensitivity vector of the proposed algorithm and the comparison algorithm. It can be see that the sensitivity of both algorithms is affected by the geometry condition.

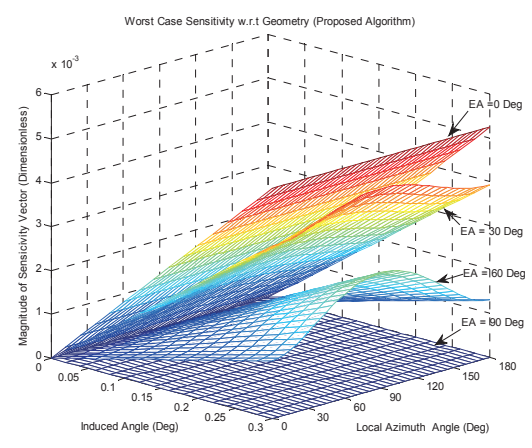

The distinct property is that the effect of the local elevation angle is different for the two algorithms. Both algorithms have a similar tendency at the local azimuth angle. However, the sensitivity of the proposed algorithm increases as elevation angle decreases, in contrast with the comparison algorithm's tendency. As described, the purpose of these algorithms is ephemeris failure detection in the absence of a verified ephemeris. This case is frequent for the first rising satellite of the day. Thus, the proposed algorithm is better because of its high sensitivity for a rising satellite with a low elevation angle. From a baseline length perspective, the proposed algorithm has high sensitivity with respect to large induced angle resulting from baseline length. The comparison algorithm has a similar tendency with baseline length; however, it has a limit on baseline length because of the basic assumption that LOS vectors of two reference station about the target satellite are quasi-parallel.[8] This means that the proposed algorithm does not have to consider the baseline length limitation and is applicable to various GNSS augmentation systems: not only the local area system (GBAS) but also widearea systems (e.g., GRAS, SBAS). The various long baseline vectors, which can be implemented with the local azimuth and local elevation angle, are required to improve detection performance of the proposed algorithm. The geometry of various azimuth angles can be implemented by multiple RSs that are not on the same line. However, this is difficult with baseline vector geometry for various local elevations, especially when based on long baseline length. Fortunately, satellites at low elevation angles are more frequent than those with high elevation angles (close to $90^{\circ}$ ) over a 24 -h

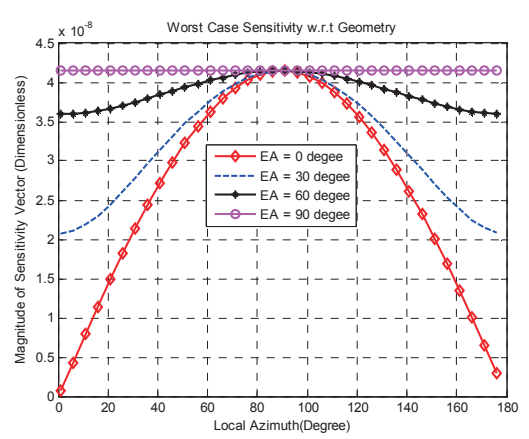

Fig. 4. Magnitude of the sensitivity vector with respect to the geometry condition

(The proposed algorithm (left) and the comparison algorithm (right)).

Table 2. Tendency of increasing sensitivity corresponding to geometry conditions.

\begin{tabular}{|l|l|l|}
\hline Geometry conditions & Comparison algorithm & Proposed algorithm \\
\hline Local elevation angle & close to $90^{\circ}$ & close to $0^{\circ}$ \\
\hline Local azimuth angle & close to $90^{\circ}$ & close to $90^{\circ}$ \\
\hline Baseline length & Increase (limited) & Increase \\
\hline
\end{tabular}


period. However, this is left to be considered in further work.

Next, we tried to verify the sensitivity equation in equation (12) for the proposed algorithm. As shown in Table 3, the various baseline vectors are composed with Suwon (SUWN), Nonsan (NONS), and Jeju (JEJU) from Seoul (SOUL) in Korea, and we use error-free range measurement to examine the influence of the geometry condition. A satellite position fault ( $1 \mathrm{~km}, \mathrm{XYZ}$ ) is imposed on all visible satellites for $24 \mathrm{~h}$.

Figure 5 shows the mean and standard deviation of test statistics with local elevation angle. As expected, the test statistics show a decreasing tendency with respect to high elevation angle.

Figure 6 shows the mean and standard deviation of test statistics with local azimuth angle. As expected, the test statistics show an increasing tendency as close to $90^{\circ}$ local azimuth angle in similar elevation angle.

Finally, long baseline length, which causes larger induced angle, can be an important factor for detection performance, as shown in both Figs. 5 and 6.

\subsection{Algorithm Realization and Availability Test using GPS Data}

In this section, we realized and evaluated the proposed algorithm using real GPS data of multiple RSs. The main items were evaluation of the correction result of the range measurement error. Then, we determined the threshold, MDE, and conducted availability testing based on the EPL at Gimpo International Airport with multiple baseline conditions in Korea.

As shown in Fig. 7, the multiple baseline conditions (6 baselines) were deployed using four reference stations operated by the National Geographic Information Institute (NGII) in Korea.

Table 3. Baseline lengths of the simulated condition.

\begin{tabular}{|l|l|l|l|}
\hline $\begin{array}{l}\text { From Seoul } \\
\text { (SOUL) }\end{array}$ & to Suwon (SUWN) & to Nonsan (NONS) & to Jeju (JEJU) \\
\hline Baseline length & $39.37 \mathrm{~km}$ & $160.18 \mathrm{~km}$ & $459.26 \mathrm{~km}$ \\
\hline
\end{tabular}
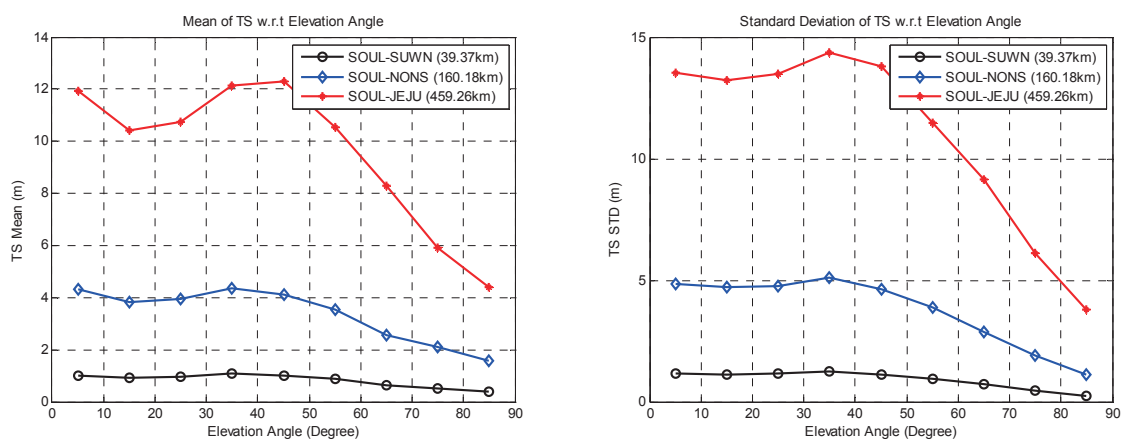

Fig. 5. Variation of test statistics in the ephemeris failure condition with respect to local elevation angle and baseline length: mean (left) and standard deviation (right).
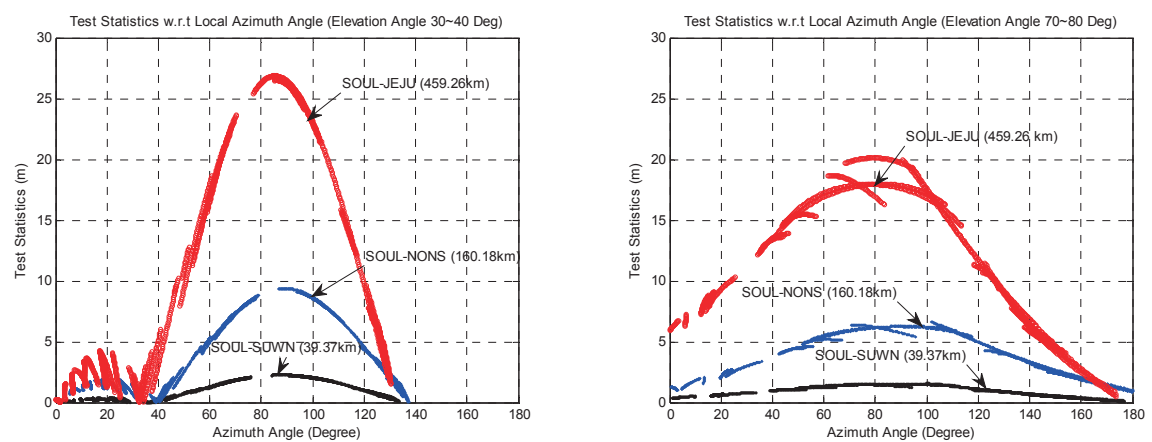

Fig. 6. Variation of test statistics in the ephemeris failure condition with respect to local azimuth angle and baseline length: in elevation angle 30$40^{\circ}$ (left) and in elevation angle $70-80^{\circ}$ (right). 
Prior to generating test statistics, we corrected the range measurement error sources, which include the ionosphere, troposphere, and satellite/receiver clock bias, as described in Section 1. Fig. 8 shows the resulting range correction error at four reference stations of all visible satellites based on 24-h data. It can be seen that the ramifications of range error sources are reduced in all visible satellites. Statistical results (mean, standard deviation, and histogram) are shown in Table 4.

To ensure reliability, many data samples are required for analysis in terms of statistics for determination of the threshold. In the present work, the mean and standard

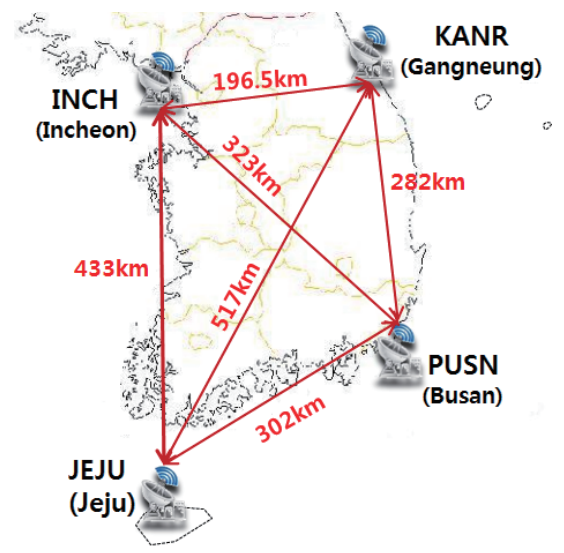

Fig. 7. Baseline construction for the simulation.

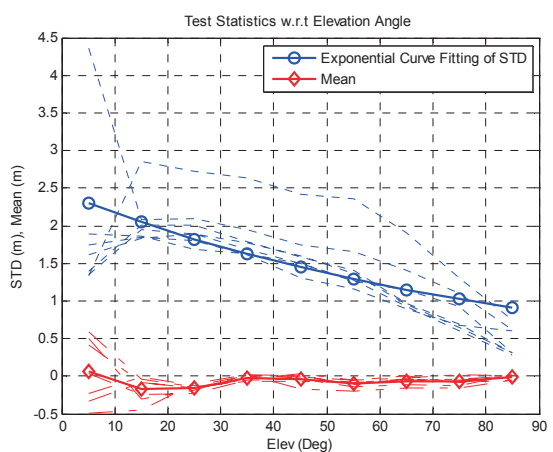

deviation of test statistics are computed using 1-week data received from 2014.9.1 to 2014.9.7 (sampling period $30 \mathrm{~s}$ ). The plot on the left hand side of Fig. 9 shows the test statistics mean (red lines with diamond markers) and standard deviation (blue lines with circular markers) with local elevation angle. A standard deviation model of test statistics is derived in equation (22). Shown in the plot on the right hand side of Fig. 9, the threshold with respect to local elevation angle is derived in equation (23), which uses the multiplier $\left(\mathrm{K}_{\mathrm{FFA}}\right)$ associated with the false alarm rate of the CAT-I requirement [12].
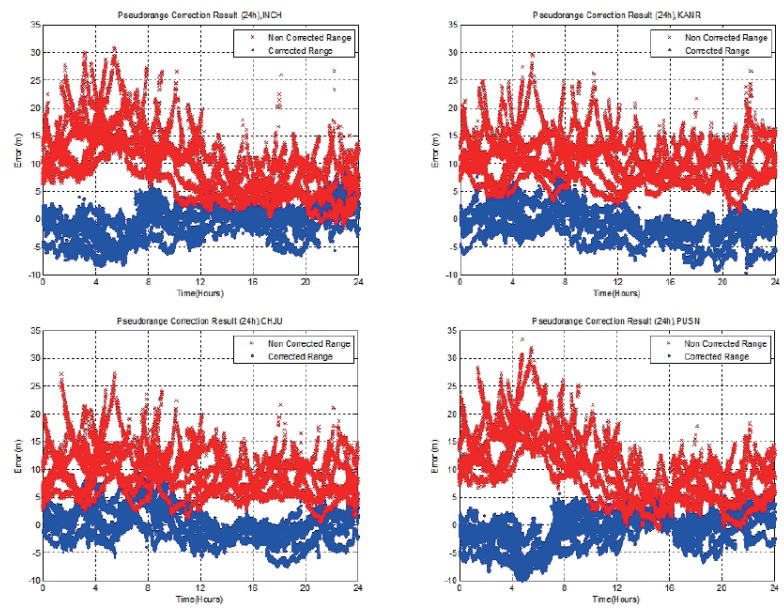

Fig. 8. Range measurement mitigation results (24 h).

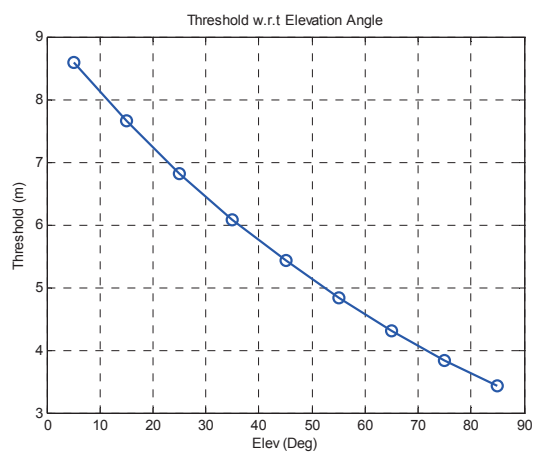

Fig. 9. Daily variation of test statistics and threshold with respect to local elevation angle.

Table 4. Range mitigation error (mean and standard deviation).

\begin{tabular}{|l|l|l|}
\hline Statistics & Mean $(\mathbf{m})$ & Standard deviation $(\mathbf{m})$ \\
\hline Before mitigation & 10.24 & 4.78 \\
\hline After mitigation & -0.56 & 2.97 \\
\hline
\end{tabular}

Table 5. False alarm probability and multiplier according to the CAT-I requirement

\begin{tabular}{|c|c|c|}
\hline Requirement & False Alarm Rate & Multiplier $\left(\mathrm{K}_{\mathrm{FFA}}\right)$ \\
\hline CAT-I & $1.9 \times 10^{-4}$ & 3.74 \\
\hline
\end{tabular}




$$
\begin{aligned}
& \sigma_{T S}^{i}(\theta)=2.431 \times e^{-0.01149 \theta} \\
& \sigma_{\text {Thresh }}^{i}(\theta)=K_{F F A} \times \sigma_{T S}^{i}(\theta)
\end{aligned}
$$

\section{$\theta$ : Elevation angle (degrees)}

Figure 10 shows an example of the test statistics and threshold on PRN 11 of the test period. In the nominal condition, we can see that the test statistic does not exceed the threshold, but does trigger a false alarm.

\subsection{MDE and Availability Testing}

The MDE of all visible satellites (24 h) is computed with equation (3-4). The missed detection probability and multiplier associated with the CAT-I requirement, as shown in Table 6, are is used to compute the MDE [18].

The left hand side of Fig. 11 shows an example of the PRN 5

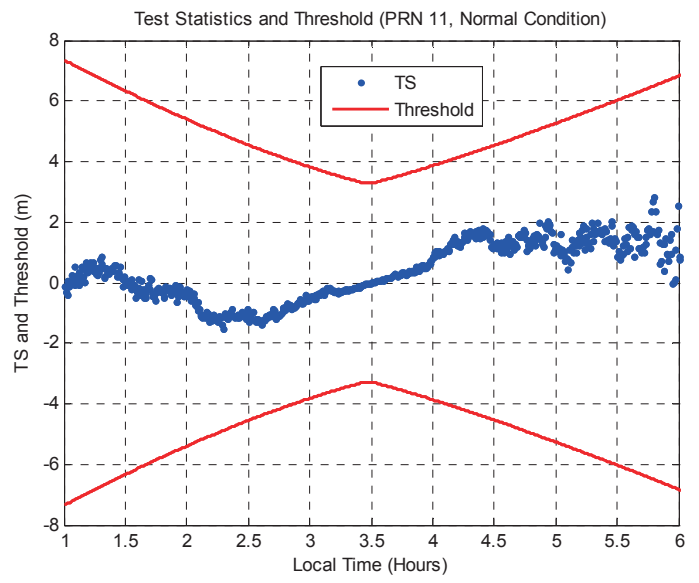

Fig. 10. Test statistics of normal condition with threshold (PRN 11)
MDE corresponding to six baselines. It is clear that the MDE of the proposed algorithm fluctuates with respect to time because of the variable worst-case sensitivity vector related to geometry of the baseline and the satellite.

Consequently, for detection performance the appropriate baseline is selected based on the least MDE value monitored among the various RS geometries in real time. The right hand side of Fig. 11 shows the least MDE value of all visible satellites and baseline conditions for a 24 -h period.

However, the figure showed unexpected MDE divergence, to be investigated further. The given satellites (PRN 1, PRN 11, PRN 26, and PRN 27) are close to highelevation-angle status. A satellite at a high elevation angle degrades the sensitivity of the algorithm and leads to an increase in the MDE value. Once again, this satellite condition is not frequent for a 1 day (24-h) period, and this algorithm focuses on rising satellites with low elevation angles. However, to ensure system integrity, the GNSS augmentation system, which implements this algorithm, should also use additional ephemeris fault detection algorithms.

Next, we present the availability result based on the ephemeris protection level that uses the MDE value. The basic simulation condition considers an aircraft trying to land at Gimpo International Airport on runway 32R (RWY 32R), located at a CAT-I decision height (DH) of $200 \mathrm{ft}$. The GPS constellation is based on 31 satellites. The reason for availability analysis at the $\mathrm{DH}$ is that the aircraft determines the use of the ground landing-aided facility at the DH (final location) associated with system requirements. The analysis period is $24 \mathrm{~h}$, considering the repeatability of the GPS constellation (approximately

Table 6. Missed detection probability and multiplier according to the CAT-I requirement

\begin{tabular}{|c|c|c|}
\hline Requirement & Probability of missed detection & Multiplier $\left(\mathrm{K}_{\mathrm{md}}\right)$ \\
\hline CAT-I & $1.0 \times 10^{-3}$ & 3.1 \\
\hline
\end{tabular}
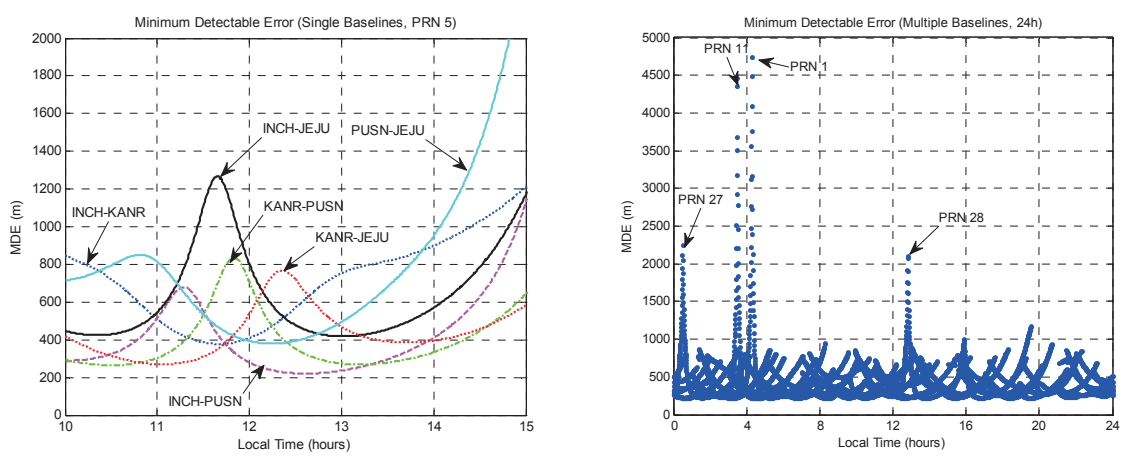

Fig. 11. MDE according to baseline vectors (PRN 5) and minimum MDE result (24 h). 
$11 \mathrm{~h} 58 \mathrm{~s}$ ) and the rotation of the earth. This property is advantageous for shortening the analysis period. The availability analysis with EPL is conducted in comparison with the same algorithm used in the sensitivity analysis in Section 3.1 [9]. The P-value of the comparison algorithm, which is used to compute the EPL, is shown in equation (24) [12].

$$
P=\left(K_{F F A}+K_{M D}\right) \times \sigma_{\phi} / b
$$

$\sigma_{\phi}$ : Standard deviation of the test statistics (double differential carrier: $0.3 \mathrm{~cm}$ )

$$
b \text { : Baseline length }(200 \mathrm{~m})
$$

where $\sigma_{\phi}$ is the noise level of the double difference carrier measurement, corresponding to an integrated multipath limiting antenna (IMLA) used for RS [8]. The value of $b$ represents the baseline length between multiple RS antennas and is similar to the RS of Gimpo International Airport.

The P-value variation over $24 \mathrm{~h}$ is shown in Fig. 12. It can be seen that the P-value of the proposed algorithm varies with time due to the geometry condition, whereas that of the comparison algorithm does not; additionally, the P-value magnitude is generally smaller for the proposed algorithm. As shown on the left side of Fig. 12, pronounced ramifications due to high-elevation-angle satellites are also observed in the P-value, as expected. Fortunately, it is still within the allowable value (CAT-I), established in ICAO Annex 10, for landing aircraft. [19].

The estimated aircraft position error due to the P-value, which represents ephemeris failure detection performance, is shown on the right side of Fig. 12. The result shown in Fig. 12 shows that position error according to P-Value is less than centimeter-scale because the effect of ephemeris failure decorrelation is small between the aircraft and the RS in the case of small displacement $(752.5 \mathrm{~m})$. However, if the displacement is larger than

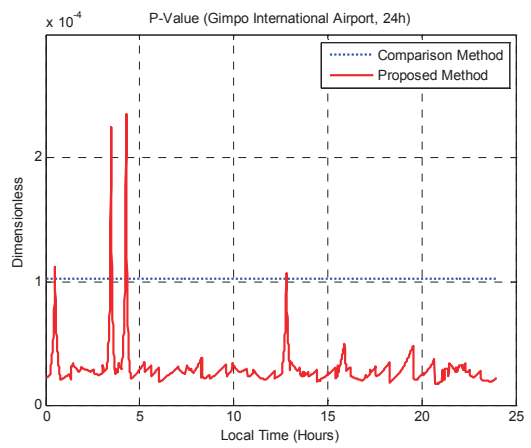

in this simulation condition, the proposed algorithm is more useful than the comparison algorithm because of the increasing of the decorrelation effect, as shown in equation (24).

The nominal error models due to airborne antenna multipath/noise (equation 25), ionosphere (equation 26), troposphere (equation 27), and RS receivers (equation 28) are applied to compute the EPL together with ephemeris failure-based position error.

$$
\begin{aligned}
& \sigma_{\text {multipath }}^{i}=0.13+0.53 e^{-\theta^{i} / 10^{\circ}}, \sigma_{\text {noise }}^{i}=a_{0, A A D}+a_{1, A A D} e^{-\theta^{i} / \theta_{c, A A D}} \\
& \sigma_{\text {iono }}^{i}=F_{p p}^{i} \times \sigma_{\text {vert_iono_gradient }} \times\left(X_{\text {air }}+2 \times \tau \times v_{\text {air }}\right) \\
& F_{p p}^{i}=\left[1-\left(\frac{R_{e} \cos \left(\theta^{i}\right)}{R_{e}+h_{I}}\right)^{2}\right] \\
& \sigma_{\text {tropo }}^{i}=\sigma_{N} h_{0} \times \frac{10^{-6}}{\sqrt{0.002+\sin ^{2}\left(\theta^{i}\right)}}\left(1-e^{-\Delta h / h_{0}}\right) \\
& \sigma_{\text {Pr_gnd }}^{\mathrm{i}}=\sqrt{\frac{\left(a_{0, G A D}+a_{1, G A D} e^{-\theta^{i} / \theta_{0, G A D}}\right)^{2}}{M}+\left(a_{2, G A D}\right)^{2}}
\end{aligned}
$$

The parameters of the given model are shown in Tables 7, 8, and 9. The airborne accuracy designator (AAD) and ground accuracy designator (GAD) are related to antenna performance index for the airborne station and the RS, respectively. [19]

Figure 13 shows the EPL result according to both algorithms. The results show that EPL will meet the availability requirement, called the alert limit (AL), established in CAT-I $(10 \mathrm{~m})$ and CAT-II/III $(4.4 \mathrm{~m})$. The effect of high elevation angle on the proposed algorithm is expected to increase the EPL (red line), but it does not threaten to exceed the AL (which would result in a loss of availability) because of considerable satellite geometry.

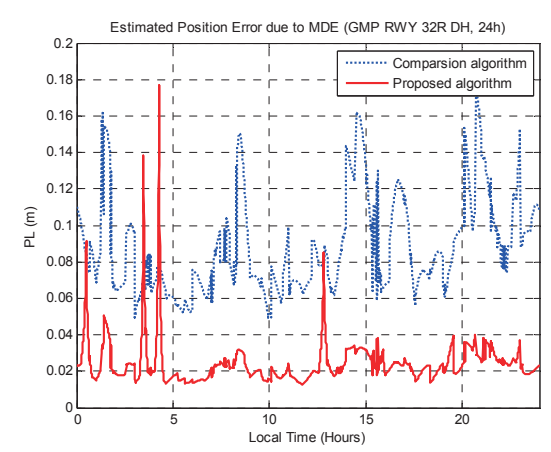

Fig. 12. P-Value (left) and estimated position error with respect to P-Value (right). 
Despite the explicit distinctions between the two algorithms, appreciable differences in EPL are not observed in Fig. 13. If the ephemeris failure decorrelation due to aircraft-RS displacement increases (as in a wide-area implementation), then the EPL based on the proposed algorithm is more relatively valuable for system availability than that of the comparison algorithm. These findings suggest that the proposed algorithm will be suitable to not only GBAS, but also SBAS and GRAS, which are implemented for wide-area applications.

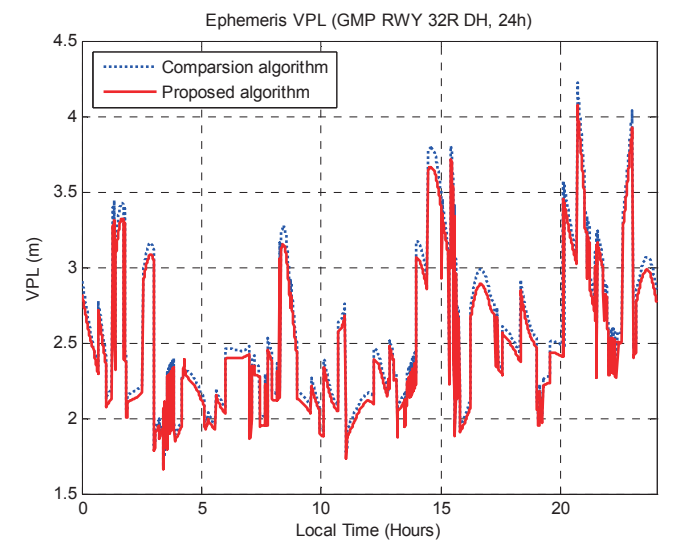

Fig. 13. EPL result at Gimpo International Airport (24 h).

\section{Conclusions and Future Work}

GNSS orbit ephemeris is broadcast to users for computing the position of a navigation satellite. The orbit ephemeris is estimated by the ground facility, which is the operational control segment (OCS) of the GPS, and affects the accuracy of the user's navigation solution. Currently, estimated performance tends to improve when using the differential GNSS (DGNSS) implementation, so ramifications of ephemeris error to the user are very small in limiting cases. However, the integrity issue has been magnified recently along with user accuracy requirements. In particular, integrity can determine system availability and any other requirements faced within the aviation community. Ephemeris constitutes a threat to integrity of the GNSS; thus, we propose an ephemeris failure detection algorithm.

Basically, the proposed algorithm uses trigonometry (the First Cosine Law) to estimate the baseline length of reference station antennas and compute the residual with true baseline length surveyed precisely. If this residual (from test statistics) exceeds a threshold value, then ephemeris failure is detected.

For detailed algorithm analysis, we introduce the generation of test statistics and a threshold value. An

Table 7. Error model parameters in the normal condition.

\begin{tabular}{|l|l|}
\hline Contents & Values \\
\hline Ionosphere slant $\left(\sigma_{\text {vert_iono_gradient }}\right)$ & $25.5 \times 10^{-6}$ \\
\hline Earth radius $\left(R_{e}\right)$ & $6378.1368 \mathrm{~km}$ \\
\hline Ionosphere thickness $\left(h_{I}\right)$ & $350 \mathrm{~km}$ \\
\hline Slant Range $\left(X_{\text {air }}\right)$ & $752.5 \mathrm{~m}$ \\
\hline Time constant $(\tau)$ & $100 \mathrm{~s}$ \\
\hline Vehicle velocity $\left(v_{\text {air }}\right)$ & $39.02 \mathrm{~m} / \mathrm{s}$ \\
\hline Troposphere scale height $\left(h_{0}\right)$ & $25,500 \mathrm{~m}$ \\
\hline Troposphere refractivity uncertainty $\left(\sigma_{N}\right)$ & 255 \\
\hline No. Reference station $(M)$ & 4 \\
\hline
\end{tabular}

Table 8. Airborne accuracy designator.

\begin{tabular}{|l|l|l|l|}
\hline AAD & $a_{0, A A D}$ & $a_{1, A A D}$ & $\theta_{C, A A D}$ \\
\hline AAD-B & 0.11 & 0.13 & 4.0 \\
\hline
\end{tabular}

Table 9. Ground accuracy designator.

\begin{tabular}{|l|l|l|l|l|l|}
\hline \multicolumn{2}{|l|}{ GAD } & $a_{0, G A D}$ & $a_{1, G A D}$ & $a_{2, G A D}$ & $\theta_{0, G A D}$ \\
\hline \multirow{2}{*}{ GAD-C } & $\theta^{i} \geq 35$ & 0.15 & 0.84 & 0.04 & 15.5 \\
\cline { 2 - 6 } & $\theta^{i}<35$ & 0.24 & 0 & 0.04 & - \\
\hline
\end{tabular}


analysis is conducted on the test statistics' sensitivity vector, corresponding to ephemeris failure, and on an availability test, based on the ephemeris protection level in Korea. The important feature is that the performance index (sensitivity and MDE) depends on geometric parameters including local elevation angle, local azimuth angle, and baseline length between the satellite and the baseline vectors. Consequently, this algorithm is more efficient for use with multiple reference stations that can generate various long baseline vectors, such as GRAS and SBAS. The availability test conducted for Gimpo International Airport in Korea was based on the EPL of a landing aircraft at CAT-I decision height. In some cases, concern is warranted by an increase in EPL (which threatens availability) because of increased MDE. However, this effect is still small enough to meet the stringent requirements (CAT-I and CAT-II/III) for precision approaches and landing.

In future work, we will address the algorithm's weakness at high elevation angles and validate it using numerous international GNSS reference data.

\section{Acknowledgement}

This work was supported partially by the Ministry of Land, Infrastructure and Transport (MOLIT) under Grant 10AVINAV01.

\section{References}

[1] B. Hoffmann-Wellenhof H. and Lichtenegger, E. Walse, 2008, GNSS; GPS, GLONASS, Galileo \& more, springer Wien New York, pp. 269 273

[2] Jeong Hwan Yang, Chang Ho Kang, Sun Young Kim, and Chan Gook Park, "International GNSS Interference Detection and Characterization Algorithm uisng AGC and Adaptive IIR Notch Filter", International Journal of Aeronautical and Space Sciences, Vol. 13, No. 4, 2012. 12, pp. 491-498

[3] Bradford W. Parkinson, et al., Global Positioning System : Theory and Applications Volume 1, AIAA, 1996, pp. 121-139

[4] Liang Heng, Grace Xingxin Gao, Todd Walter, and Per Enge, "GPS Ephemeris Error Screening and Results for 2006-2009", Proceedings of the 2010 International Technical Meeting of the Institute of Navigation (ION ITM 2010), San Diego, CA, Jan 2010, pp. 1014-1022

[5] Liang Heng, Grace Xingxin Gao, Todd Walter, and Per Enge: "GPS signal-in-space anomalies in the last decade: Data mining of 400,000,000 GPS navigation messages,"
Proceedings of the 23rd International Technical Meeting of the Satellite Division of the Institute of Navigation (ION GNSS 2010), Portland, OR, Sep 2010, pp. 3115-3122

[6] Haochen Tang, Sam Pullen, Per Enge, Livio Gratton, Boris Pervan, Mats Brenner, Joe Scheitlin, and Paul Kline, "Ephemeris Type A Fault Analysis and Mitigation for LAAS", Proceedings of Position Location and Navigation Symposium (PLANS), 2010 IEEE/ION, 4-6 May 2010, pp. 654-666

[7] Gang Xie, Optimal On-airport Monitoring of the Integrity of GPS-Based Landing System, Ph.D Thesis, Stanford University, March 2004, pp. 39-43.

[8] Boris Pervan, Livio Gratton, “Orbit Ephemeris Monitors for Local Area Differential GPS”, Journal of IEEE Transactions on Aerospace and Electronic Systems, Vol. 41, No. 2, April 2005.

[9] Jiyun Lee, Sam Pullen, Boris Pervan, and Livio Gratton, "Monitoring Global Positioning Satellite Orbit Errors for Aircraft Landing System”, Journal of Aircraft, Vol. 43, No. 3, May-June 2006.

[10] Boris Pervan, and Fang Cheng Chan, "Detecting Global Positioning System Orbit Errors Using Short-Baseline Carrier-Phase Measurements" Journal of Guidance, Control, and Dynamics, Vol. 26, No. 1, January-February 2003.

[11] Gang Xie, Optimal On-airport Monitoring of the Integrity of GPS-Based Landing System, Ph.D Thesis, Stanford University, March 2004, pp. 26-43.

[12] Pullen S., Lee J, Luo, M., Pervan, B, Chan, F-C, and Gratton, L., "Ephemeris Protection Level Equation and Monitor Algorithm for GBAS", Proceedings of the 14th International Technical Meeting of the Satellite Division of the Institute of Navigation, Salt Lak City, UT, Sept, 11-14, 2001.

[13] Chan, F.C., Detection of Global Positioning Satellite Orbit Errors Using Short-Baseline Carrier Phase Measurements, M.S.Thesis, Dept. of Mechanical, Materials, and Aerospace Engineering, Illinois Inst. of Technology, Chicago, Aug. 2001, pp. 17-19.

[14] Jongsun Ahn, Hyang-Sig Jun, Chan-Hong Yeom, Sangkyung Sung, and Young Jae Lee, "Sensitivity Analysis of Ephemeris Fault Detection Algorithm based on Baseline Length Estimation Method", Proceeding of KGS 2014, Nov. 2014, pp. 693-696.

[15] Jongsun Ahn, Eunsung Lee, Moon Beom Heo, Sangkyung Sung, and Young Jae Lee, "Research of MDE of for Baseline-based GPS Ephemeris Fault Detection Algorithm", Proceeding of KSAS 2014, Nov. 2014, pp. 330-332.

[16] Mastumoto, S, Pullen, S, Rotkowitz, M., and Pervan, B., "GPS Ephemeris Verification for Local Area Augmentation System (LAAS) Ground Station," Proceedings of the 12th International Technical Meeting of the Satellite Division of the Institute of Navigation, Inst., of Navigation, Alexandria, VA, 
Jongsun Ahn Orbit Ephemeris Failure Detection in a GNSS Regional Application

1999. 691-703.

[17] Specification Category 1 Local Area Augmentation System Non-Federal Ground Facility, U.S. Federal Aviation Administration, FAA/AND 710-2937, Washington, DC, May, 2001

[18] Shively. C, "LAAS Integrity Risk due to satellite ephemeris faults", Proceedings of the 14th International Technical Meeting of the Satellite Division of the Institute of Navigation, Salt Lake City, UT, Sept. 11-14, 2001.

[19] Draft, ICAO Annex 10, GBAS CAT II/III Development Baseline SARPs, 17-29, May 2010 meeting of the Navigation System Panel (NSP) Working Group 